\title{
The community-curated Pristionchus pacificus genome facilitates automated gene annotation improvement in related nematodes
}

\author{
Christian Rödelsperger(1)
}

\begin{abstract}
Background: The nematode Pristionchus pacificus is an established model organism for comparative studies with Caenorhabditis elegans. Over the past years, it developed into an independent animal model organism for elucidating the genetic basis of phenotypic plasticity. Community-based curations were employed recently to improve the quality of gene annotations of $P$. pacificus and to more easily facilitate reverse genetic studies using candidate genes from C. elegans.

Results: Here, I demonstrate that the reannotation of phylogenomic data from nine related nematode species using the community-curated P. pacificus gene set as homology data substantially improves the quality of gene annotations. Benchmarking of universal single copy orthologs (BUSCO) estimates a median completeness of 84\% which corresponds to a 9\% increase over previous annotations. Nevertheless, the ability to infer gene models based on homology already drops beyond the genus level reflecting the rapid evolution of nematode lineages. This also indicates that the highly curated C. elegans genome is not optimally suited for annotating non-Caenorhabditis genomes based on homology. Furthermore, comparative genomic analysis of apparently missing BUSCO genes indicates a failure of ortholog detection by the BUSCO pipeline due to the insufficient sample size and phylogenetic breadth of the underlying OrthoDB data set. As a consequence, the quality of multiple divergent nematode genomes might be underestimated.
\end{abstract}

Conclusions: This study highlights the need for optimizing gene annotation protocols and it demonstrates the benefit of a high quality genome for phylogenomic data of related species.

Keywords: Comparative genomics, Evolution, Phylogeny, Parasite, Caenorhabditis elegans, BUSCO, PPCAC

\section{Background}

Genome sequencing efforts across all domains of life have broadened our understanding about how phenotypic novelty coincides with genomic innovations. This was facilitated by continuous improvement of sequencing technology during the last two decades, which

\footnotetext{
Correspondence: christian.roedelsperger@tuebingen.mpg.de Department for Integrative Evolutionary Biology, Max Planck Institute for Developmental Biology, Max-Planck-Ring 9, 72076 Tübingen, Germany
}

allowed the generation of high-quality genome assemblies in large-scale phylogenomic contexts. In contrast, gene annotation protocols have evolved at a much slower rate [1]. One reason for the slow progress in the further developments of gene annotation protocols was likely the absence of universal benchmarking standards. Even though gene predictions were often evaluated against available expression data, such results were difficult to compare between organisms due to strong

(c) The Author(s). 2021 Open Access This article is licensed under a Creative Commons Attribution 4.0 International License, which permits use, sharing, adaptation, distribution and reproduction in any medium or format, as long as you give appropriate credit to the original author(s) and the source, provide a link to the Creative Commons licence, and indicate if changes were made. The images or other third party material in this article are included in the article's Creative Commons licence, unless indicated otherwise in a credit line to the material. If material is not included in the article's Creative Commons licence and your intended use is not permitted by statutory regulation or exceeds the permitted use, you will need to obtain permission directly from the copyright holder. To view a copy of this licence, visit http://creativecommons.org/licenses/by/4.0/ The Creative Commons Public Domain Dedication waiver (http://creativecommons.org/publicdomain/zero/1.0/) applies to the data made available in this article, unless otherwise stated in a credit line to the data. 
differences in transcriptomic resources across various genome projects. This changed when alternative approaches, such as benchmarking universal single copy orthologs (BUSCO) [2], employed comparative genomics to define a set of highly conserved orthologous genes that should be expected in a given genome assembly. While the BUSCO completeness level has become a widely used quality measure with similar importance as the N50 measure for assembly contiguity, its informative value is highly dependent on the quality and sampling of the underlying orthology data, which may differ vastly across taxonomic groups. Currently, multiple representative genomes of the highly diverse and rapidly evolving nematode phylum are still poorly annotated. To overcome this problem in the case of the nematode model organism Pristionchus pacificus, community-based curations have recently been initiated to improve the quality of gene annotations $[3,4]$. P. pacificus was initially established for comparative studies with the classical nematode model organism Caenorhabditis elegans [5], but more recently it gained importance as an independent model system for elucidating the genetic basis of phenotypic plasticity [6-8] and the emergence of novel genes [9-11]. P. pacificus has a chromosome-scale genome assembly and computationally generated gene annotations based on transcriptomic data, protein homology data, and gene predictions were of relatively high quality [12]. However, further strand-specific RNA-seq and Iso-seq data pointed towards the presence of numerous artificial gene fusions in gene dense regions of the genome [3]. This motivated a screen for suspicious gene models based on comparative genomic approaches and to propose corrections after manual inspection by community annotators. Two rounds of community curations improved the BUSCO completeness level from 86 to 98\% (nematode odb9 data set) [2-4].

Here, I make use of the community-curated annotations of the $P$. pacificus genome to improve the annotations of related Pristionchus and other genomes of the family Diplogastridae, which were recently sequenced to study the evolutionary dynamics of novel gene families [9]. This demonstrates that a single high quality reference data set is sufficient to improve gene annotations in related genomes.

\section{Results}

High quality gene annotations are rare outside the Caenorhabditis clade

In order to assess the current status of nematode genome quality, I analyzed gene annotations from 54 nematode species as obtained from WormBase ParaSite (version WBPS14) using the BUSCO approach, which tests for the presence of highly conserved single copy orthologs [2, 13]. Using an arbitrary cutoff of $>80 \%$
BUSCO completeness of single copy genes to define high quality gene annotations, this analysis shows that high quality gene annotations are rare outside the Caenorhabditis clade (Fig. 1). Few exceptions are the genomes of the free-living Oscheius tipulae and the parasitic Haemonchus contortus [18], Dirofilaria immitis [19], Loa loa [20], Brugia malayi [21], and Onchocerca volvulus [22]. Please note that the most recent updates of the $P$. pacificus gene annotations have not yet been integrated into WormBase, which explains why the BUSCO completeness is shown at around $80 \%$ (Fig. 1). As BUSCO genes are defined as genes that should be present as single copy in at least $90 \%$ of genomes, the low completeness values point towards substantial annotation problems in various genomes (Fig. 1). Alternatively, these discrepancies could reflect true cases of gene losses in specific lineages. Another explanation could be that divergent orthologs may not be detected, as the underlying nematode odb10 data set only contains seven nematode species (Fig. 1) and these do not represent the full range of genomic diversity of the nematode phylum [14, 23]. Despite these alternative explanations, the phylum-wide assessment of annotation quality based on BUSCO completeness strongly suggests substantial need for improvement, which is in accordance with complementary studies assessing the quality of multiple nematode genomes $[24,25]$.

\section{A single high quality reference set is sufficient to improve gene annotations in related species}

Previously, comparative genomic screens were combined with community curation to improve the quality of gene annotations in P. pacificus [4]. Here, the objective is to demonstrate how community curation of a single genome can be used to automatically improve the quality of phylogenomic data from related species. To this end, I reannotated nine nematode genomes of the family Diplogastridae including seven other Pristionchus species, which were sequenced previously as part of a phylogenomic study to investigate the evolutionary dynamics of novel gene families $[9,26]$. Specifically, predicted open reading frames (ORFs) in assembled RNAseq transcripts [27] as well as protein sequences of the community-curated P. pacificus annotation (El Paco gene annotation, version 3) were mapped to the draft genomes with the help of the exonerate alignment tool [28]. Subsequently, a simple heuristic was applied to pick only one representative gene model per locus (see Methods, Additional file 1, Figure S1). The raw mappings of transcribed ORFs, the previous and the newly generated gene annotations were then evaluated based on the level of BUSCO completeness (nematode odb10 data set). To assess, to what extent the manually improved $P$. pacificus data set yielded better gene 


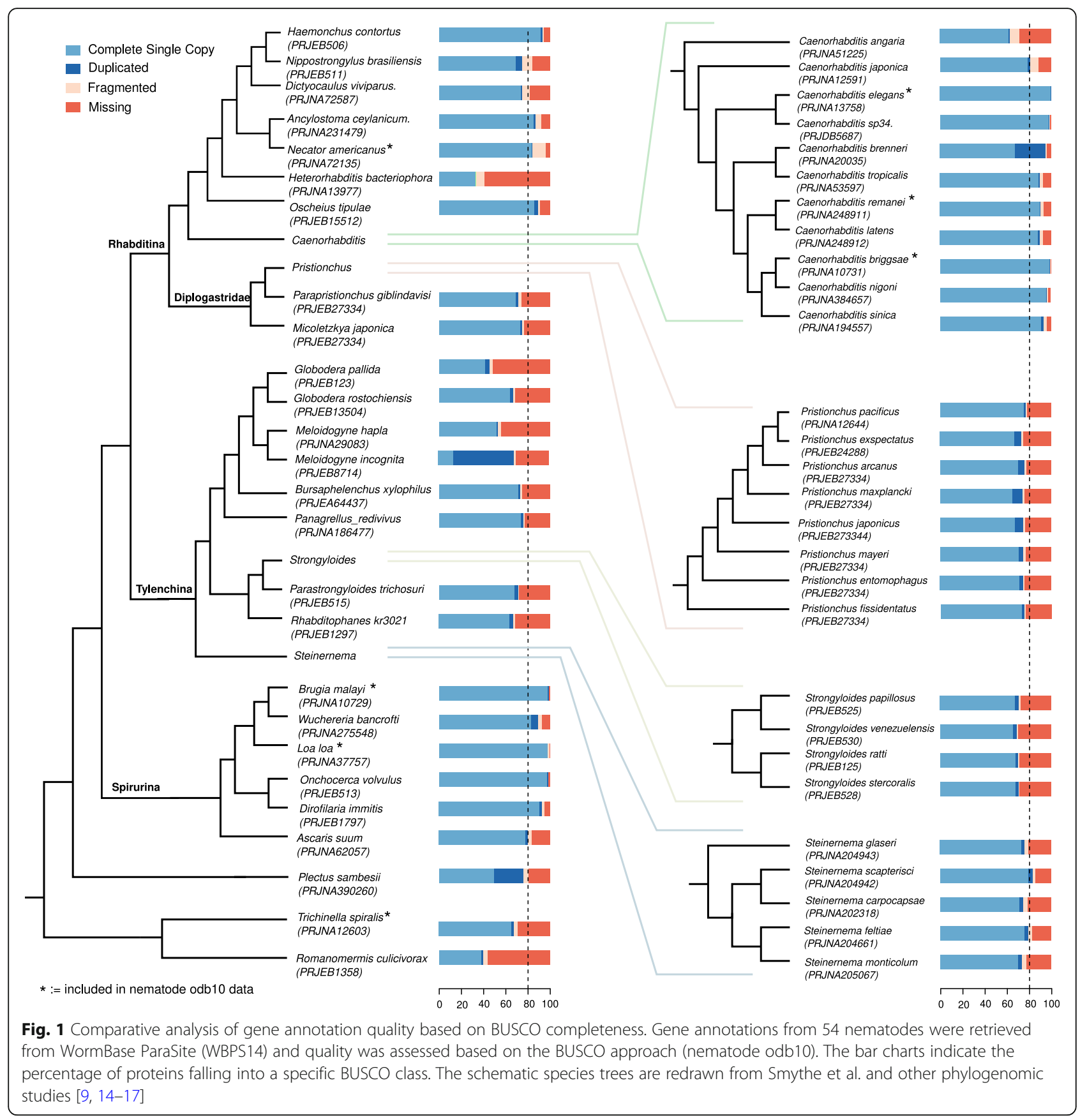

annotations of related nematodes, the same reannotation procedure was applied using the automatically generated $P$. pacificus gene annotations as homology data [12]. Similarly, the gain in gene annotation accuracy by having a more closely related reference data set was evaluated by comparison with the highly curated but evolutionary distant C. elegans annotation. For the nine diplogastrid genomes, reannotation with the community-curated $P$. pacificus genome yielded a median BUSCO completeness of $84 \%$ (Fig. 2a, Table 1), which corresponds to a median improvement of $9 \%$ over the previous version of gene annotations [9]. The genome of Parapristionchus giblindavisi is the only exception where the new P. pacificus annotations did not result in an improved gene annotation (Fig. 2a). Most likely, this is due to general problems with this assembly, as it has the lowest level of contiguity, a high fraction of ambiguous bases, and the highest ratio of incorrectly oriented read pairs in realignment analysis [9]. Such problems potentially arose from remaining heterozygosity [29] and might impair accurate gene annotation. However, all other genomes showed that using the community-curated $P$. pacificus data set 


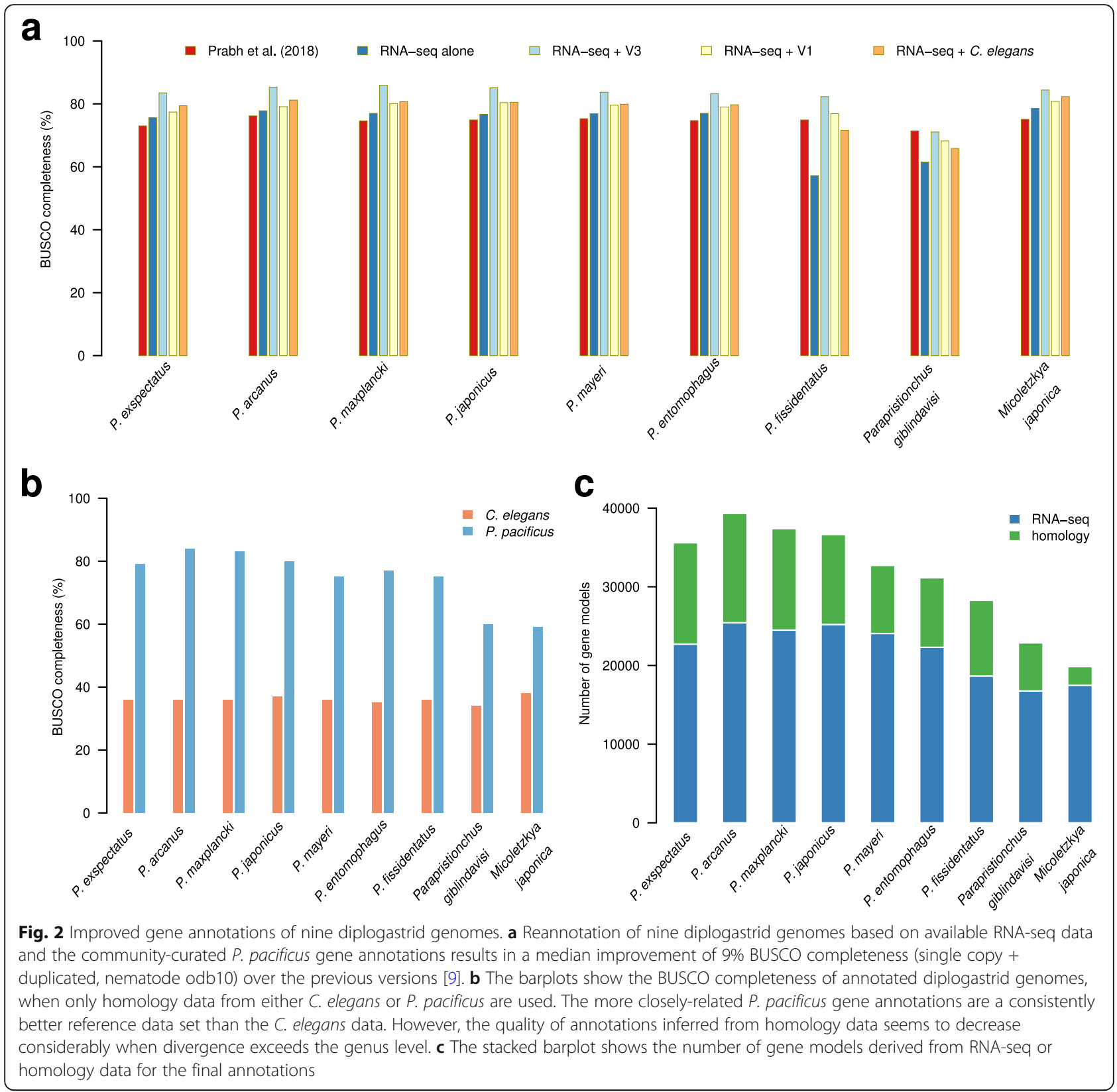

as homology data yielded the most complete gene annotations even when compared to the highly curated but evolutionary distant C. elegans data set (Fig. 2a).

\section{Divergence across genera hinders gene model inference from homology data}

The fact that homology data from $P$. pacificus yields better results than C. elegans data indicates that the ability to transfer gene annotations across species drops with increasing sequence divergence. This would imply that taking the highly curated C. elegans data as a reference, is suboptimal for annotating divergent nematode genomes. To test if the drop in homology-based annotation accuracy occurs already within the same nematode family, I reevaluated the BUSCO completeness of the gene annotations only inferred from homology data (Fig. 2b). While gene models based on homology with C. elegans have a constantly low BUSCO completeness of around $36 \%$, the completeness values for the gene models inferred from community-curated P. pacificus data range between 59 and $84 \%$ (Fig. 2b). Note that the two non-Pristionchus genomes have with 59 and $60 \%$ the lowest completeness values whereas the lowest value for the remaining Pristionchus genomes is $75 \%$ (Fig. 2b). Moreover, the evaluation of the contribution of homology-inferred vs. RNA-seq derived gene 
Table 1 Assembly and gene annotation features of diplogastrid genomes

\begin{tabular}{|c|c|c|c|c|c|c|}
\hline \multirow[t]{2}{*}{ Species } & \multicolumn{3}{|l|}{ Prabh et al. 2018} & \multicolumn{3}{|l|}{ This study } \\
\hline & Number of gene models & $\begin{array}{l}\text { BUSCO odb9 } \\
\text { C/D/F/M (\%) }\end{array}$ & $\begin{array}{l}\text { BUSCO odb10 } \\
\text { C/D/F/M (\%) }\end{array}$ & Number of gene models & $\begin{array}{l}\text { BUSCO odb9 } \\
\text { C/D/F/M (\%) }\end{array}$ & $\begin{array}{l}\text { BUSCO odb10 } \\
\text { C/D/F/M (\%) }\end{array}$ \\
\hline P. exspectatus & 31,172 & $79 / 7 / 6 / 8$ & $67 / 6 / 2 / 25$ & 35,595 & $85 / 7 / 5 / 4$ & $77 / 7 / 2 / 14$ \\
\hline P. arcanus & 35,909 & $81 / 7 / 4 / 8$ & $71 / 6 / 2 / 22$ & 39,331 & $86 / 8 / 3 / 2$ & 79/7/2/13 \\
\hline P. maxplancki & 31,765 & $75 / 11 / 7 / 8$ & $65 / 7 / 2 / 23$ & 37,393 & $80 / 13 / 4 / 2$ & $74 / 12 / 2 / 12$ \\
\hline P. japonicus & 31,996 & $77 / 8 / 6 / 10$ & $68 / 7 / 2 / 23$ & 36,638 & $84 / 9 / 4 / 4$ & $76 / 9 / 2 / 13$ \\
\hline P. mayeri & 36,554 & $81 / 5 / 6 / 8$ & $71 / 4 / 2 / 23$ & 32,719 & $86 / 5 / 6 / 4$ & $79 / 4 / 3 / 14$ \\
\hline P. entomophagus & 37,279 & $82 / 4 / 6 / 8$ & $71 / 3 / 1 / 24$ & 31,150 & $87 / 4 / 5 / 4$ & $79 / 4 / 2 / 15$ \\
\hline P. fissidentatus & 25,634 & $83 / 2 / 6 / 10$ & $73 / 2 / 2 / 24$ & 28,283 & $87 / 2 / 7 / 4$ & $80 / 3 / 3 / 15$ \\
\hline P. giblindavisi & 35,770 & $74 / 3 / 10 / 13$ & $69 / 2 / 3 / 26$ & 22,872 & 70/3/14/13 & $68 / 3 / 6 / 23$ \\
\hline M. japonica & 24,971 & $79 / 2 / 9 / 9$ & $73 / 2 / 2 / 23$ & 19,855 & $88 / 2 / 5 / 5$ & $82 / 2 / 2 / 13$ \\
\hline
\end{tabular}

The table shows a comparison between the previous [9] and the current gene annotations for nine diplogastrid genomes. The number of gene models is denoted together with the BUSCO results for the odb9 $(N=982$ orthologs) and odb10 ( $N=3131$ orthologs). C Complete single copy, $D$ Duplicated, $F$ Fragmented, M Missing

models to the final gene annotations also show a drop in the contribution of homology data for the two non-Pristionchus species (Fig. 2c). Taken together, these results suggest that the ability to transfer gene models based on protein conservation already drops beyond genus-level sequence divergence. This strongly limits the usefulness of model organism data such as from $C$. elegans to be helpful for annotating genomes in evolutionary distant nematode clades.

\section{The quality of several nematode genomes might be underestimated}

The new gene annotations of the nine diplogastrid genomes contain between 19 and 39 thousand gene models that are completely evidence-based as they are either supported by transcriptional evidence or by protein conservation with $P$. pacificus (Table 1). However, between 9 and 15\% of BUSCO genes (nematode odb10 data set) seem to be missing in the Pristionchus genomes (Table 1). If these missing genes are due to misannotations in individual genomes, further rounds of manual corrections across this phylogenomic data set could be used to reannotate missing genes in selected genomes. In such a case, the abundance of gene absence/presence patterns should be more or less randomly distributed. However, if missing genes are due to massive gene losses across the diplogastrid lineage, the distribution of patterns should be dominated by a phylogenetic pattern that could be parsimoniously explained by a single evolutionary event (Fig. 3a). The two most abundant presence/absence patterns are 1490 BUSCO genes that are found in all nine diplogastrid genomes and 316 genes that seem to be missing in the Parapristionchus giblindavisi genome, which seems to be the most problematic data set (Fig. 3a). The third most abundant pattern arises from 204 genes that were not found in any of the diplogastrid genomes, which is suggestive of a lineagespecific gene loss. To exclude that these genes are missing due to gene annotation failure, the BUSCO pipeline was run in genome mode against the raw assembly (Additional file 1, Table S1). This confirmed that 185 (91\%) of those genes could not be detected by running BUSCO on the raw genome assembly. As mentioned above, an alternative explanation would be that these genes are present but could not be detected by the BUSCO pipeline as the nematode odb10 data set does not represent the full diversity of the nematode phylum $[14,23]$. Thus, running the BUSCO pipeline with a phylogenetically more broadly sampled set of taxa should capture these missing genes. Consistently, the older nematode odb9 data set, which included data from $P$. pacificus, yields higher completeness values (median value $91 \%$, Table 1 ). To further test the possibility of undetected orthologs, I used a complementary approach to find one-to-one orthologs of the corresponding C. elegans genes in the diplogastrid genomes based on best reciprocal BLASTP searches. This revealed that 101 (50\%) of these 204 genes have predicted one-to-one orthologs in all diplogastrid genomes, which points to a failure of detection of the BUSCO pipeline (Fig. 3b). Comparison of the bitscores from BLASTP searches between BUSCO genes in C. elegans and their putative orthologs in the diplogastrid genomes shows a pronounced difference between the diplogastrid sequences that were detected as orthologs by BUSCO and the sequences that were only identified as best-reciprocal hits (Fig. 4a). Further analysis of alignment length and percentage identity indicates that this difference is due to stronger sequence divergence in the diplogastrid sequences that were not identified as orthologs by BUSCO (Fig. 4b, c). Similarly, the analysis of length-normalized bitscores shows strong differences between BUSCO orthologs and best- 


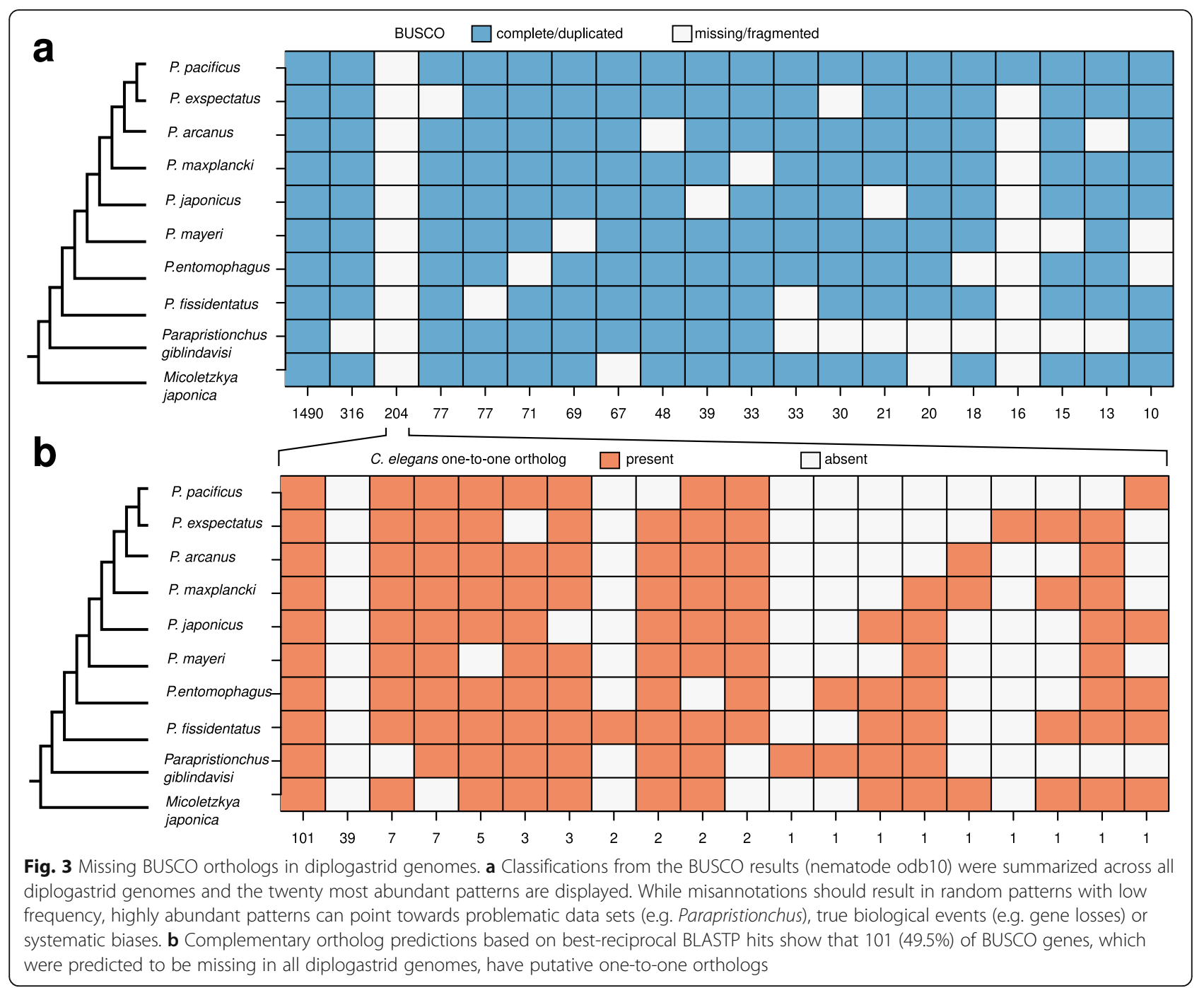

reciprocal hits, whereas the overall aligned proportion is more comparable between both groups (Additional file 1, Figure S2). To further support that at least some of these genes are truly orthologs, I reconstructed gene trees for 12 randomly chosen candidate gene families (Fig. 5a-1). Most of these phylogenies largely resemble the species phylogeny (Fig. 1) and therefore support the one-to-one orthology relationship. Thus, I conclude that the insufficient sample size and phylogenetic breadth of the nematode odb10 data set may cause failures in the ortholog detection by the BUSCO pipeline and that the quality of divergent nematode genomes might therefore be underestimated.

\section{Discussion}

In this study, I have demonstrated the benefit of the community-curated $P$. pacificus gene annotations for phylogenomic data of related species. The automated improvements of gene annotations in other diplogastrid nematodes suggest that not every genome has to be manually curated, but that a single high-quality genome per genus or family is sufficient to allow effective gene model inference based on homology. It has to be noted that community curation is not the only way to obtain high quality gene annotations as previous studies showed that high levels of BUSCO completeness could be obtained by extensively optimized application of gene prediction tools such as BRAKER and AUGUSTUS [25, 30, 31]. Specifically, the training of gene models is the most important step for such ab initio gene prediction methods. However, the question how good gene annotations have to be will depend on the research topics that are going to be studied. Previous studies of novel gene origin in P. pacificus pointed towards an inflated number of species-specific orphan genes that are most likely gene prediction artifacts recognizing spurious coding signals on the antisense strand of truly protein-coding genes [10]. Thus, to facilitate more conclusive studies of 

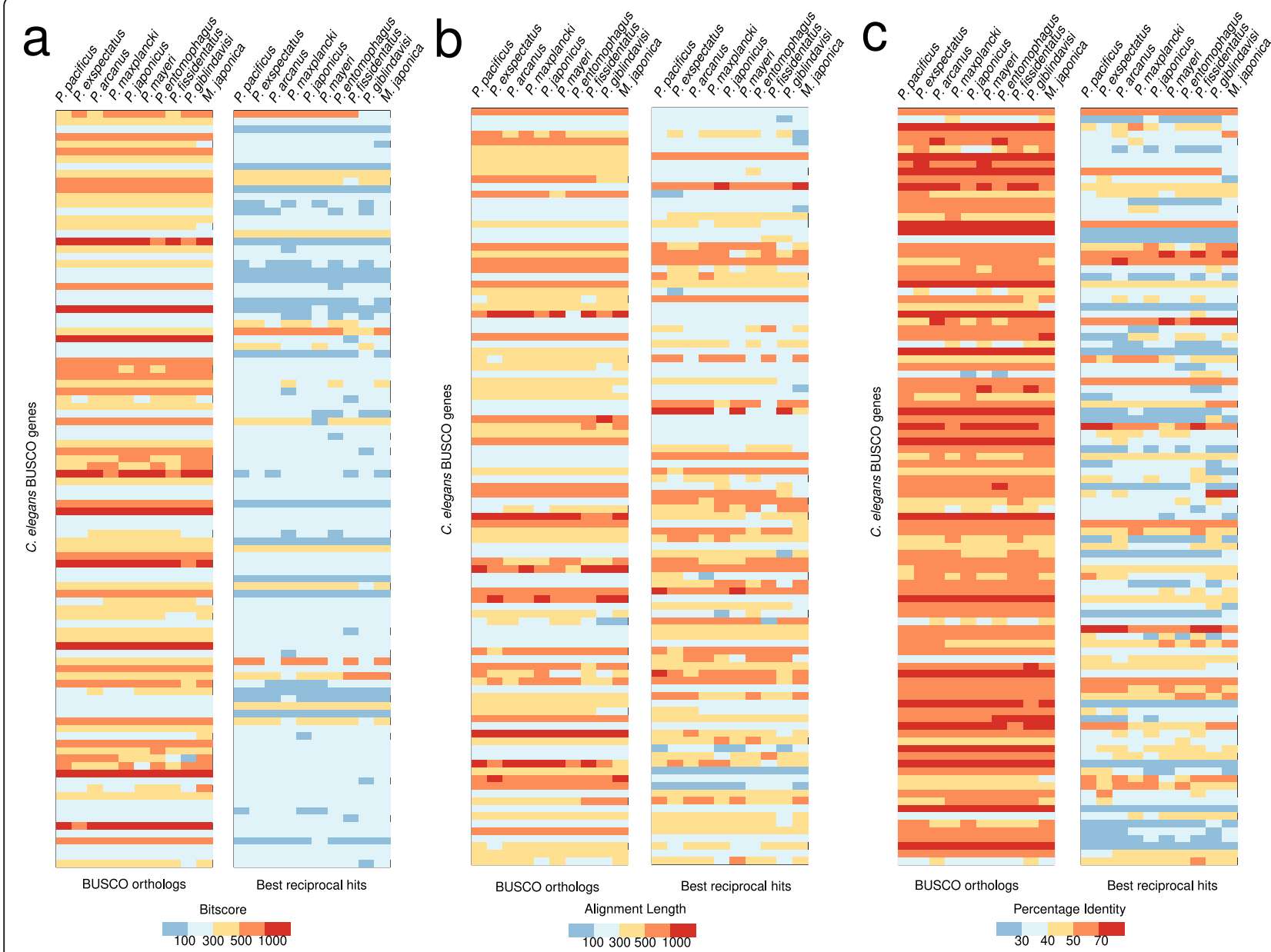

Fig. 4 Sequence divergence causes orthology detection failures. a The left heatmap shows the bitscores for 101 randomly subsampled BUSCO orthologs derived from a BLASTP search of the C. elegans proteins against annotated protein sets of the ten diplogastrid genomes. The right heatmap shows the bitscores for 101 putative orthologs that were only predicted based on best-reciprocal BLASTP hits. $\mathbf{b}$ The heatmaps show the BLASTP alignment lengths for BUSCO genes from C. elegans against diplogastrid proteins for 101 randomly subsampled genes with BUSCO orthologs (left) and for 101 best-reciprocal hits (right). c The heatmaps show the percentage identity between BUSCO genes from C. elegans and their putative diplogastrid orthologs

gene birth processes using purely evidence-based set of gene annotations, species-specific genes without RNAseq support were removed from current annotations [4]. Furthermore, other types of gene prediction artifacts such as artificially fused gene models do not necessarily impact BUSCO completeness levels but impair the detection of orthologs [3]. In this context, community annotation seems to be one of the most effective methods to increase gene annotation quality beyond what can be achieved using automated pipelines. For example, in the case of the nine diplogastrid genomes, BUSCO genes that are found in the genome or transcriptome (Additional file 1, Tables S1, S2), but not in the final gene annotations can be taken as candidates to further improve gene annotation quality by manual curation. Nevertheless, it has to be noted that the effectiveness of community annotation is highly dependent on the availability of abundant transcriptomic resources. Thus, requiring support by either transcriptome data or protein conservation for every gene is not generally feasible for genome projects of phylogenetically isolated species with limited expression data.

The drop in the ability to infer gene models based on homology beyond the genus level emphasizes the rapid evolution and extreme diversity of nematode lineages $[32,33]$. This might also at least partially contribute to the failure of detection of 101 putative orthologs by the BUSCO pipeline, as the underlying OrthoDB data set (nematode odb10) appears to be too small and unevenly sampled to capture the full diversity of the nematode phylum [14, 23]. As a consequence, the quality of many nematode genomes might be underestimated. In the case of the diplogastrid genomes, the completeness level is likely underestimated by up to 3\% (101 out of 3131 


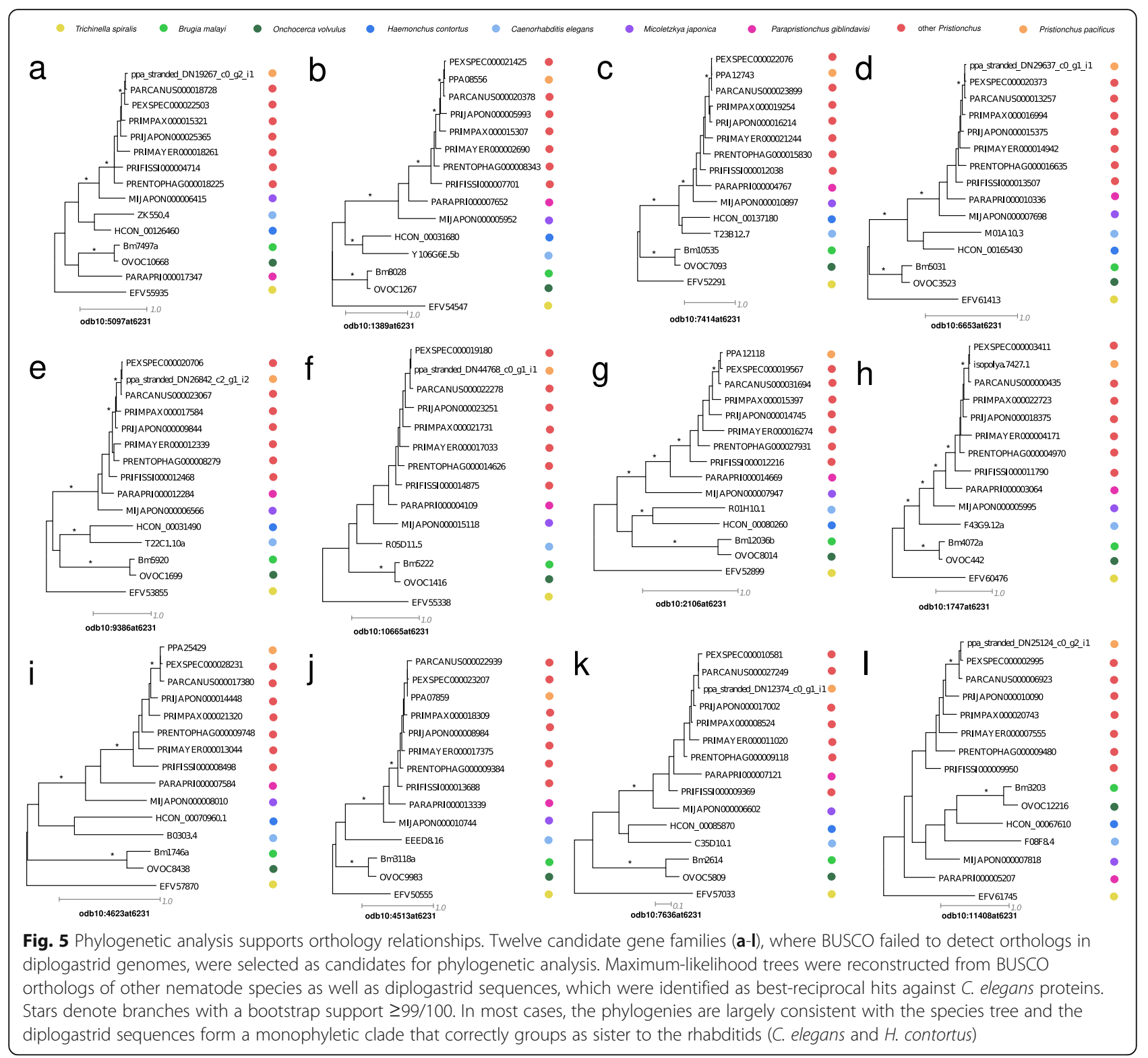

BUSCO genes that were not detected but are present in all 10 genomes). The fact that this problem seems to be less pronounced in the older odb9 data set (Table 1) suggests that the more recent odb10 data set is not well suited for assessing the genome quality of divergent nematode species.

Thus, my study not only highlights the need to optimize gene annotation pipelines, but also points at potential problems in the benchmarking processes. Further work will be needed to establish more comprehensive benchmarking data sets as well as to optimize annotation protocols. For example, apart from the alignment program exonerate [28], there are multiple alternative approaches for transferring gene models such as GMAP or liftover, and Liftoff [34,35]. In addition, gene models from transcriptome and homology data were integrated into a non-redundant gene set using a heuristic approach that selects the gene model with longest ORF per $100 \mathrm{bp}$ window. Hereby, genes with less than three exons or a predicted protein length of less than 60 amino acids were discarded. Such arbitrary thresholds may remove unusually short genes with important biological functions and might not be directly transferable to other nematode genomes with a smaller number of exons per gene than P. pacificus [36]. Apart from the mentioned caveats, the presented methods should be directly applicable to genomes of other taxonomic groups, even outside of nematodes.

In future, gene model inference based on the highly curated $P$. pacificus data set will help other genomic 
studies in this clade of nematodes. Currently, the genus Pristionchus has around 50 described species and genuswide phylogenetic studies revealed interesting trends such as the parallel emergence of hermaphroditism and the convergent evolution of specific pheromones [27, 37]. The generation of high quality genomes of further members of the Pristionchus genus may therefore help to characterize and compare the genomic basis of these convergent patterns. Simultaneously, the pool of more than thousand $P$. pacificus strains was successfully exploited to dissect the genetic basis of phenotypic variation at a population level $[38,39]$. Such unbiased genetic screens revealed that genomic changes including gene duplication and loss cause natural variation in pheromone production and response [40, 41]. Thus, properly annotated de novo assemblies of different $P$. pacificus strains will greatly aid the interpretation of associations between genotypes and phenotypes and thus complement future genetic screens in P. pacificus.

\section{Conclusions}

While genome sequencing technologies have undergone tremendous development over the past 20 years, gene annotation protocols evolved at a much slower rate. In the case of the nematode model organism Pristionchus pacificus, community-based gene curations have previously been presented as an effective means to lift annotation quality above the level of what could be obtained by automated pipelines. Here, I make use of these community-curated annotations to automatically improve phylogenomic data of nine related nematodes. This work has three major conclusions. First, the community-curated $P$. pacificus genome improves the completeness of related nematode genomes by a median of $9 \%$ over previous annotations. With BUSCO completeness levels between 83 and $86 \%$, the reannotated Pristionchus genomes are more complete than most other members of the nematode phylum. Second, the ability to infer gene models based on homology already drops beyond the genus level, which implies that the highly curated C. elegans data is not well suited for annotation of divergent nematode genomes. Third, the insufficient sample size and phylogenetic breadth of the BUSCO and OrthoDB data sets may prohibit the detection of orthologs and thus cause an underestimation of nematode genome quality.

\section{Methods}

\section{Generation of evidence-based gene annotations}

The data set of transcribed ORFs was taken from a previous study where transcriptomes of different Diplogastrid species were assembled from mixed-stage RNA-seq data (Additional file 1, Table S2) and partial and complete ORFs with a minimal length of 40 amino acids were extracted [27]. These transcribed ORFs were aligned against the respective reference assembly with the help of the exonerate protein2genome program with the following parameter settings: --bestn 2, --dnawordlen 20, and --maxintron 20,000 (version 2.2.0) [28]. The homology model data set was generated by aligning P. pacificus proteins (El Paco gene annotation, version 3) against the reference assemblies using exonerate with the same parameter settings [4]. The -bestn option was set to two in order to annotate potential duplicates. Homology models and transcribed ORFs were merged into a joint annotation which included possibly multiple gene models (different isoforms in the assembled transcripts, different evidence types) for a given gene. The complexity of the joint annotation was reduced by a simple heuristic to generate a set of non-redundant annotations. First, gene models from each DNA strand were separated and every start and end coordinate of an exon was assigned to a $100 \mathrm{bp}$ window. Second, For each 100 bp window the coverage was computed as the number of features that were assigned to this window. Third, starting with the most highly covered $100 \mathrm{bp}$ window, the intersecting gene model with the longest ORF was chosen whereas all other intersecting gene models were discarded. At this step only gene models with ORFs of at least 60 amino acids and at least three exons were considered. This last step was successively executed for all other windows. As gene models in P. pacificus tend to have more exons per gene than most other nematodes [36], the threshold of at least three exons per genes was implemented to prevent an inflation of gene counts by partially assembled transcript fragments and transcriptional noise. The source code for generating these gene annotations is written in perl. All scripts have been compiled in a software package called PPCAC and are available at https://github.com/roedelsberg/ppcac/.

\section{Comparative genomic analysis}

Protein sequences from 54 nematodes were retrieved from WormBase ParaSite (WBPS14). In case of multiple isoforms, the longest isoform was chosen as the representative sequence [20,33, 42-58]. To assess the completeness level of gene annotations, the BUSCO pipeline (version 4.1.1) was run in protein mode with the nematode odb10 data set (Creation date: 2019-11-20, number of species: 7, number of BUSCOs: 3131). This combination of BUSCO and odb10 was used for most analyses (Figs. 1, 2 and 3). However, for comparisons with a set of orthologous genes, which include data from $P$. pacificus, the BUSCO pipeline (version 3.0.1) was run with the nematode odb9 data set (Creation date: 2016-02-13, number of species: 8 , number of BUSCOs: 982). From the result files of the BUSCO pipeline, genes that were classified to be missing were extracted and compared 
with predicted one-to-one orthologs for C. elegans genes that were obtained by best-reciprocal BLASTP searches (version 2.6.0, e-value $<0.0001$, bitscores were used to define the best BLASTP hit). From the BLASTP results, bitscores, percentage identity, and alignment lengths were extracted and used for comparison between the BUSCO orthologs and best-reciprocal hits. For visualization and comparison of BLASTP features, the set of 1490 BUSCO genes with orthologs in all diplogastrid genomes (Fig. 3a) was repeatedly downsampled to match the 101 putative orthologs that were identified by best-reciprocal BLASTP searches (Fig. 3b). Phylogenies of selected orthologous groups were generated by extracting the corresponding BUSCO orthologs in the high quality gene annotations of C. elegans, Haemonchus contortus, Brugia malayi, Onchocerca volvulus, and Trichinella spiralis and combining these with the bestreciprocal hits in the diplogastrid genomes. Subsequently, protein sequences were aligned by the MUSCLE program (version 3.8.31, default options) and maximum likelihood trees were calculated using the phangorn package in R (version 3.4.4, LG substitution model with optimization of base frequencies and invariant sites, 100 bootstrap pseudoreplicates) $[59,60]$. The orthologous $T$. spiralis sequences, as identified by BUSCO pipeline, were used to root the trees.

\section{Abbreviations}

BUSCO: Benchmarking Universal Single Copy Orthologs; ORF: Open Reading Frame; PPCAC: Perl Package for Customized Annotation Computing

\section{Supplementary Information}

The online version contains supplementary material available at https://doi. org/10.1186/s12864-021-07529-x.

Additional file 1: Figure S1. Heuristic approach for reducing the complexity of redundant annotations. A $30 \mathrm{~kb}$ genomic locus is visualized in the Integrative Genomics Viewer with different tracks showing the exonerate alignments of protein homology data, transcribed ORFs, and the resulting non-redundant gene annotations. The lower plot shows the coverage of exonic features in 100bp windows. First, a 100bp window with maximal coverage is selected. Second, the overlapping gene model with longest ORF is chosen as representative gene model for this locus. Third, all other overlapping gene models are excluded from further analysis. Fourth, the next 100bp window is chosen. This procedure is continued until all 100bp windows have been processed. The final gene models are shown in the track labeled as 'Non-redundant gene models'. Figure S2. Comparison of normalized bitscores and aligned proportion. a The left heatmap shows the normalized bitscores (bitscore / alignment length) for 1490 BUSCO orthologs derived from a BLASTP search of the C. elegans proteins against annotated protein sets of the ten diplogastrid genomes. The central heatmap shows the data for 101 randomly subsampled BUSCO orthologs. The right heatmap shows the normalized bitscore for 101 putative orthologs that were only predicted based on bestreciprocal BLASTP hits. b The aligned proportion was computed as the length of the BLASTP alignment divided by the protein length of the C. elegans query sequence. The heatmaps show the aligned proportion of all BUSCO orthologs, randomly subsampled BUSCO orthologs, and bestreciprocal BLASTP hits. Table S1. Summary of genome assemblies. Basic features of nine diplogastrid genomes are shown together with the BUSCO results for the OrthoDB data sets odb10 and odb9 (C:= Complete single copy, D:= Duplicated, F:= Fragmented, M:=Missing - Percentage values are presented as integers. Therefore, values might not always sum up to 100). Table S2. Summary of transcriptome assemblies. Basic features of nine transcriptome assemblies are shown together with the BUSCO results for the OrthoDB data sets odb10 and odb9 (C:= Complete single copy, D:= Duplicated, F:= Fragmented, M:=Missing - Percentage values are presented as integers. Therefore, values might not always sum up to 100).

\section{Acknowledgements}

I would like to thank the whole Pristionchus community for their long-term interest in studying $P$. pacificus and thus motivating this work. I would also like to thank three anonymous reviewers for very helpful comments on the manuscript.

\section{Author's contributions}

CR performed analyses and wrote the manuscript. The author read and approved the final manuscript.

\section{Funding}

This work was funded by the Max Planck Society. The funding bodies played no role in the design of the study and collection, analysis, and interpretation of data and in writing the manuscript. Open Access funding enabled and organized by Projekt DEAL.

\section{Availability of data and materials}

Genome data is available at the European Nucleotide Archive under the study accession PRJEB27334. All data sets were submitted to WormBase ParaSite and are also available at http://pristionchus.org/download/ diplogastrid_annotation_ppcac_v1.tgz. Source code is available at https:// github.com/roedelsberg/ppcac.

\section{Declarations}

Ethics approval and consent to participate

Not applicable.

\section{Consent for publication}

Not applicable.

\section{Competing interests}

The author declares that he has no competing interests.

Received: 9 November 2020 Accepted: 12 March 2021

Published online: 25 March 2021

\section{References}

1. Salzberg SL. Next-generation genome annotation: we still struggle to get it right. Genome Biol. 2019;20(1):92. https://doi.org/10.1186/s13059-019-1715-2.

2. Simão FA, Waterhouse RM, loannidis P, Kriventseva EV, Zdobnov EM. BUSCO: assessing genome assembly and annotation completeness with single-copy orthologs. Bioinformatics. 2015;31(19):3210-2. https://doi.org/1 0.1093/bioinformatics/btv351.

3. Rödelsperger C, Athanasouli M, Lenuzzi M, Theska T, Sun S, Dardiry M, Wighard S, Hu W, Sharma DR, Han Z. Crowdsourcing and the feasibility of manual gene annotation: a pilot study in the nematode Pristionchus pacificus. Sci Rep. 2019;9(1):18789. https://doi.org/10.1038/s41598-019-553 59-5.

4. Athanasouli M, Witte H, Weiler C, Loschko T, Eberhardt G, Sommer RJ, Rödelsperger C. Comparative genomics and community curation further improve gene annotations in the nematode Pristionchus pacificus. BMC Genomics. 2020;21(1):708. https://doi.org/10.1186/s12864-020-07100-0.

5. Sternberg PW. Why Caenorhabditis elegans is great and Pristionchus pacificus might be better. In: Pristionchus pacificus; 2015. p. 1-17. https:// doi.org/10.1163/9789004260306_002.

6. Sieriebriennikov B, Prabh N, Dardiry M, Witte H, Röseler W, Kieninger MR, et al. A developmental switch generating phenotypic plasticity is part of a conserved multi-gene locus. Cell Rep. 2018;23:2835-43.e4.

7. Sieriebriennikov B, Sun S, Lightfoot JW, Witte H, Moreno E, Rödelsperger C, Sommer RJ. Conserved nuclear hormone receptors controlling a novel 
plastic trait target fast-evolving genes expressed in a single cell. PLoS Genet. 2020;16(4):e1008687. https://doi.org/10.1371/journal.pgen.1008687.

8. Sommer RJ. Phenotypic plasticity: from theory and genetics to current and future challenges. Genetics. 2020;215(1):1-13. https://doi.org/10.1534/ genetics.120.303163.

9. Prabh N, Roeseler W, Witte H, Eberhardt G, Sommer RJ, Rödelsperger C. Deep taxon sampling reveals the evolutionary dynamics of novel gene families in nematodes. Genome Res. 2018;28(11):1664-74. https://doi.org/1 0.1101/gr.234971.118.

10. Prabh N, Rödelsperger C. De Novo, Divergence, and Mixed Origin Contribute to the Emergence of Orphan Genes in Nematodes. G3. 2019;9: 2277-86.

11. Rödelsperger C, Ebbing A, Sharma DR, Okumura M, Sommer RJ, Korswagen HC. Spatial Transcriptomics of nematodes identifies sperm cells as a source of genomic novelty and rapid evolution. Mol Biol Evol. 2021;38(1):229-43. https://doi.org/10.1093/molbev/msaa207.

12. Rödelsperger C, Meyer JM, Prabh N, Lanz C, Bemm F, Sommer RJ. Singlemolecule sequencing reveals the chromosome-scale genomic architecture of the nematode model organism Pristionchus pacificus. Cell Rep. 2017; 21(3):834-44. https://doi.org/10.1016/j.celrep.2017.09.077.

13. Howe KL, Bolt BJ, Shafie M, Kersey P, Berriman M. WormBase ParaSite - a comprehensive resource for helminth genomics. Mol Biochem Parasitol. 2017;215:2-10. https://doi.org/10.1016/.molbiopara.2016.11.005.

14. Smythe $A B$, Holovachov O, Kocot KM. Improved phylogenomic sampling of free-living nematodes enhances resolution of higher-level nematode phylogeny. BMC Evol Biol. 2019;19(1):121. https://doi.org/10.1186/s12862-01 9-1444-X.

15. Stevens L, Félix M-A, Beltran T, Braendle C, Caurcel C, Fausett S, Fitch D, Frézal L, Gosse C, Kaur T, Kiontke K, Newton MD, Noble LM, Richaud A, Rockman MV, Sudhaus W, Blaxter M. Comparative genomics of 10 new Caenorhabditis species. Evol Lett. 2019;3(2):217-36. https://doi.org/10.1002/ evl3.110.

16. Hunt VL, Tsai IJ, Coghlan A, Reid AJ, Holroyd N, Foth BJ, Tracey A, Cotton JA, Stanley EJ, Beasley H, Bennett HM, Brooks K, Harsha B, Kajitani R, Kulkarni A, Harbecke D, Nagayasu E, Nichol S, Ogura Y, Quail MA, Randle N, Xia D, Brattig NW, Soblik H, Ribeiro DM, Sanchez-Flores A, Hayashi T, Itoh T, Denver DR, Grant W, Stoltzfus JD, Lok JB, Murayama H, Wastling J, Streit A, Kikuchi T, Viney M, Berriman M. The genomic basis of parasitism in the Strongyloides clade of nematodes. Nat Genet. 2016;48(3):299-307. https://doi.org/10.1038/ ng.3495.

17. Dillman AR, Macchietto M, Porter CF, Rogers A, Williams B, Antoshechkin I, Lee MM, Goodwin Z, Lu X, Lewis EE, Goodrich-Blair H, Stock SP, Adams BJ, Sternberg PW, Mortazavi A. Comparative genomics of Steinernema reveals deeply conserved gene regulatory networks. Genome Biol. 2015;16(1):200. https://doi.org/10.1186/s13059-015-0746-6.

18. Doyle SR, Tracey A, Laing R, Holroyd N, Bartley D, Bazant W, Beasley H, Beech R, Britton C, Brooks K, Chaudhry U, Maitland K, Martinelli A, Noonan $J D$, Paulini M, Quail MA, Redman E, Rodgers FH, Sallé G, Shabbir MZ, Sankaranarayanan G, Wit J, Howe KL, Sargison N, Devaney E, Berriman M, Gilleard JS, Cotton JA. Genomic and transcriptomic variation defines the chromosome-scale assembly of Haemonchus contortus, a model gastrointestinal worm. Commun Biol. 2020;3(1):656. https://doi.org/10.1038/ s42003-020-01377-3.

19. Godel C, Kumar S, Koutsovoulos G, Ludin P, Nilsson D, Comandatore F, Wrobel N, Thompson M, Schmid CD, Goto S, Bringaud F, Wolstenholme A, Bandi C, Epe C, Kaminsky R, Blaxter M, Mäser P. The genome of the heartworm, Dirofilaria immitis, reveals drug and vaccine targets. FASEB J. 2012;26(11):4650-61. https://doi.org/10.1096/fj.12-205096

20. Desjardins CA, Cerqueira GC, Goldberg JM, Dunning Hotopp JC, Haas BJ, Zucker J, et al. Genomics of Loa loa, a Wolbachia-free filarial parasite of humans. Nat Genet. 2013;45(5):495-500. https://doi.org/10.1038/ng.2585.

21. Foster JM, Grote A, Mattick J, Tracey A, Tsai Y-C, Chung M, Cotton JA, Clark TA, Geber A, Holroyd N, Korlach J, Li Y, Libro S, Lustigman S, Michalski ML, Paulini M, Rogers MB, Teigen L, Twaddle A, Welch L, Berriman M, Dunning Hotopp JC, Ghedin E. Sex chromosome evolution in parasitic nematodes of humans. Nat Commun. 2020;11(1):1964. https://doi.org/10.1038/s41467-02 0-15654-6.

22. Cotton JA, Bennuru S, Grote A, Harsha B, Tracey A, Beech R, Doyle SR, Dunn M, Hotopp JCD, Holroyd N, Kikuchi T, Lambert O, Mhashilkar A, Mutowo P, Nursimulu N, Ribeiro JMC, Rogers MB, Stanley E, Swapna LS, Tsai IJ, Unnasch TR, Voronin D, Parkinson J, Nutman TB, Ghedin E, Berriman M, Lustigman S.
The genome of Onchocerca volvulus, agent of river blindness. Nat Microbiol. 2017;2(2). https://doi.org/10.1038/nmicrobiol.2016.216.

23. Kriventseva EV, Kuznetsov D, Tegenfeldt F, Manni M, Dias R, Simão FA, Zdobnov EM. OrthoDB v10: sampling the diversity of animal, plant, fungal, protist, bacterial and viral genomes for evolutionary and functional annotations of orthologs. Nucleic Acids Res. 2019;47(D1):D807-11. https:// doi.org/10.1093/nar/gky1053.

24. Gilabert A, Curran DM, Harvey SC, Wasmuth JD. Expanding the view on the evolution of the nematode dauer signalling pathways: refinement through gene gain and pathway co-option. BMC Genomics. 2016;17(1):476. https:// doi.org/10.1186/s12864-016-2770-7.

25. McLean F, Berger D, Laetsch DR, Schwartz HT, Blaxter M. Improving the annotation of the Heterorhabditis bacteriophora genome. Gigascience. 2018;7(4). https://doi.org/10.1093/gigascience/giy034.

26. Rödelsperger C, Neher RA, Weller AM, Eberhardt G, Witte H, Mayer WE, Dieterich C, Sommer RJ. Characterization of genetic diversity in the nematode Pristionchus pacificus from population-scale resequencing data. Genetics. 2014;196(4):1153-65. https://doi.org/10.1534/genetics.113.159855.

27. Rödelsperger C, Röseler W, Prabh N, Yoshida K, Weiler C, Herrmann M, et al. Phylotranscriptomics of Pristionchus Nematodes reveals parallel gene loss in six hermaphroditic lineages. Curr Biol. 2018;28:3123-7.e5.

28. Slater GSC, Birney E. Automated generation of heuristics for biological sequence comparison. BMC Bioinformatics. 2005;6(1):31. https://doi.org/1 0.1186/1471-2105-6-31.

29. Barrière A, Yang S-P, Pekarek E, Thomas CG, Haag ES, Ruvinsky I. Detecting heterozygosity in shotgun genome assemblies: lessons from obligately outcrossing nematodes. Genome Res. 2009;19(3):470-80. https://doi.org/1 $0.1101 / \mathrm{gr} .081851 .108$.

30. Hoff KJ, Lange S, Lomsadze A, Borodovsky M, Stanke M. BRAKER1: unsupervised RNA-Seq-based genome annotation with GeneMark-ET and AUGUSTUS. Bioinformatics. 2016;32(5):767-9. https://doi.org/10.1093/ bioinformatics/btv661.

31. Casasa S, Biddle JF, Koutsovoulos GD, Ragsdale EJ. Polyphenism of a novel trait integrated rapidly evolving genes into ancestrally plastic networks. Mol Biol Evol. 2020;38(2):331-43. https://doi.org/10.1093/molbev/msaa235.

32. Telford MJ, Copley RR. Improving animal phylogenies with genomic data. Trends Genet. 2011;27(5):186-95. https://doi.org/10.1016/j.tig.2011.02.003.

33. Consortium IHG, International Helminth Genomes Consortium. Comparative genomics of the major parasitic worms. Nat Genet. 2019;51(1):163-74. https://doi.org/10.1038/s41588-018-0262-1.

34. Wu TD, Watanabe CK. GMAP: a genomic mapping and alignment program for mRNA and EST sequences. Bioinformatics. 2005;21(9):1859-75. https:// doi.org/10.1093/bioinformatics/bti310.

35. Shumate A, Salzberg SL. Liftoff: accurate mapping of gene annotations. Bioinformatics. 2020. https://doi.org/10.1093/bioinformatics/btaa1016.

36. Rödelsperger C, Streit A, Sommer RJ. Structure, function and evolution of the nematode genome. eLS. 2013. https://doi.org/10.1002/9780470015902.a 0024603.

37. Dong C, Weadick CJ, Truffault V, Sommer RJ. Convergent evolution of small molecule pheromones in Pristionchus nematodes. eLife. 2020;9. https://doi. org/10.7554/elife.55687.

38. McGaughran A, Rödelsperger C, Grimm DG, Meyer JM, Moreno E, Morgan K, Leaver M, Serobyan V, Rakitsch B, Borgwardt KM, Sommer RJ. Genomic profiles of diversification and genotype-phenotype Association in Island Nematode Lineages. Mol Biol Evol. 2016;33(9):2257-72. https://doi.org/10.1 093/molbev/msw093.

39. Lightfoot JW, Wilecki M, Rödelsperger C, Moreno E, Susoy V, Witte H, Sommer RJ. Small peptide-mediated self-recognition prevents cannibalism in predatory nematodes. Science. 2019;364(6435):86-9. https://doi.org/1 0.1126/science.aav9856.

40. Falcke JM, Bose N, Artyukhin AB, Rödelsperger C, Markov GV, Yim JJ, et al. Linking Genomic and Metabolomic Natural Variation Uncovers Nematode Pheromone Biosynthesis. Cell Chem Biol. 2018;25:787-96.e12.

41. Mayer MG, Rödelsperger C, Witte H, Riebesell M, Sommer RJ. The orphan gene dauerless regulates Dauer development and intraspecific competition in nematodes by copy number variation. PLoS Genet. 2015;11(6):e1005146. https://doi.org/10.1371/journal.pgen.1005146.

42. Opperman CH, Bird DM, Williamson VM, Rokhsar DS, Burke M, Cohn J, Cromer J, Diener S, Gajan J, Graham S, Houfek TD, Liu Q, Mitros T, Schaff J, Schaffer R, Scholl E, Sosinski BR, Thomas VP, Windham E. Sequence and genetic map of Meloidogyne hapla: a compact nematode genome for 
plant parasitism. Proc Natl Acad Sci U S A. 2008;105(39):14802-7. https://doi. org/10.1073/pnas.0805946105.

43. Abad P, Gouzy J, Aury J-M, Castagnone-Sereno P, Danchin EGJ, Deleury E, Perfus-Barbeoch L, Anthouard V, Artiguenave F, Blok VC, Caillaud MC, Coutinho PM, Dasilva C, de Luca F, Deau F, Esquibet M, Flutre T, Goldstone JV, Hamamouch N, Hewezi T, Jaillon O, Jubin C, Leonetti P, Magliano M, Maier TR, Markov GV, McVeigh P, Pesole G, Poulain J, Robinson-Rechavi M, Sallet E, Ségurens B, Steinbach D, Tytgat T, Ugarte E, van Ghelder C, Veronico P, Baum TJ, Blaxter M, Bleve-Zacheo T, Davis EL, Ewbank JJ, Favery B, Grenier E, Henrissat B, Jones JT, Laudet V, Maule AG, Quesneville H, Rosso MN, Schiex T, Smant G, Weissenbach J, Wincker P. Genome sequence of the metazoan plant-parasitic nematode Meloidogyne incognita. Nat Biotechnol. 2008;26(8):909-15. https://doi.org/10.1038/nbt.1482.

44. McNulty SN, Strübe C, Rosa BA, Martin JC, Tyagi R, Choi Y-J, et al. Dictyocaulus viviparus genome, variome and transcriptome elucidate lungworm biology and support future intervention. Sci Rep. 2016;6(1):20316. https://doi.org/10.1038/srep20316.

45. Schwarz EM, Hu Y, Antoshechkin I, Miller MM, Sternberg PW, Aroian RV. The genome and transcriptome of the zoonotic hookworm Ancylostoma ceylanicum identify infection-specific gene families. Nat Genet. 2015;47(4): 416-22. https://doi.org/10.1038/ng.3237.

46. Tang YT, Gao X, Rosa BA, Abubucker S, Hallsworth-Pepin K, Martin J, Tyagi R, Heizer E, Zhang X, Bhonagiri-Palsikar V, Minx P, Warren WC, Wang Q, Zhan B, Hotez PJ, Sternberg PW, Dougall A, Gaze ST, Mulvenna J, Sotillo J, Ranganathan S, Rabelo EM, Wilson RK, Felgner PL, Bethony J, Hawdon JM, Gasser RB, Loukas A, Mitreva M. Genome of the human hookworm Necator americanus. Nat Genet. 2014;46(3):261-9. https://doi.org/10.1038/ng.2875.

47. Bai X, Adams BJ, Ciche TA, Clifton S, Gaugler R, Kim K-S, Spieth J, Sternbers PW, Wilson RK, Grewal PS. A lover and a fighter: the genome sequence of an entomopathogenic nematode Heterorhabditis bacteriophora. PLoS One. 2013;8(7):e69618. https://doi.org/10.1371/journal.pone.0069618.

48. Cotton JA, Lilley CJ, Jones LM, Kikuchi T, Reid AJ, Thorpe P, Tsai IJ, Beasley H, Blok V, Cock PJ, Eves-van den Akker S, Holroyd N, Hunt M, Mantelin S, Naghra H, Pain A, Palomares-Rius JE, Zarowiecki M, Berriman M, Jones JT, Urwin PE. The genome and life-stage specific transcriptomes of Globodera pallida elucidate key aspects of plant parasitism by a cyst nematode. Genome Biol. 2014;15(3):R43. https://doi.org/10.1186/gb-2014-15-3-r43,

49. Eves-van den Akker S, Laetsch DR, Thorpe P, Lilley CJ, Danchin EG, Da Rocha $\mathrm{M}$, et al. The genome of the yellow potato cyst nematode, Globodera rostochiensis, reveals insights into the basis of parasitism and virulence. Genome Biol. 2016;17:124.

50. Kikuchi T, Cotton JA, Dalzell JJ, Hasegawa K, Kanzaki N, McVeigh P, Takanashi T, Tsai IJ, Assefa SA, Cock PJA, Otto TD, Hunt M, Reid AJ, SanchezFlores A, Tsuchihara K, Yokoi T, Larsson MC, Miwa J, Maule AG, Sahashi N, Jones JT, Berriman M. Genomic insights into the origin of parasitism in the emerging plant pathogen Bursaphelenchus xylophilus. PLoS Pathog. 2011; 7(9):e1002219. https://doi.org/10.1371/journal.ppat.1002219.

51. Srinivasan J, Dillman AR, Macchietto MG, Heikkinen L, Lakso M, Fracchia KM, Antoshechkin I, Mortazavi A, Wong G, Sternberg PW. The draft genome and transcriptome of Panagrellus redivivus are shaped by the harsh demands of a free-living lifestyle. Genetics. 2013;193(4):1279-95. https://doi.org/10.1534/ genetics.112.148809.

52. Small ST, Reimer $L$, Tisch DJ, King CL, Christensen BM, Siba PM, Kazura JW, Serre D, Zimmerman PA. Population genomics of the filarial nematode parasite Wuchereria bancrofti from mosquitoes. Mol Ecol. 2016;25(7):146577. https://doi.org/10.1111/mec.13574.

53. Wang J, Mitreva M, Berriman M, Thorne A, Magrini V, Koutsovoulos G, Kumar S, Blaxter ML, Davis RE. Silencing of germline-expressed genes by DNA elimination in somatic cells. Dev Cell. 2012;23(5):1072-80. https://doi. org/10.1016/.j.devcel.2012.09.020.

54. Beltran T, Barroso C, Birkle TY, Stevens L, Schwartz HT, Sternberg PW, et al. Comparative Epigenomics reveals that RNA Polymerase II pausing and Chromatin Domain Organization Control Nematode piRNA Biogenesis. Dev Cell. 2019;48:793-810.e6.

55. Mitreva M, Jasmer DP, Zarlenga DS, Wang Z, Abubucker S, Martin J, Taylor CM, Yin Y, Fulton L, Minx P, Yang SP, Warren WC, Fulton RS, Bhonagiri V, Zhang X, Hallsworth-Pepin K, Clifton SW, McCarter JP, Appleton J, Mardis ER, Wilson RK. The draft genome of the parasitic nematode Trichinella spiralis. Nat Genet. 2011;43(3):228-35. https://doi.org/10.1038/ng.769.

56. Schiffer PH, Kroiher M, Kraus C, Koutsovoulos GD, Kumar S, Camps JIR, et al. The genome of Romanomermis culicivorax: revealing fundamental changes in the core developmental genetic toolkit in Nematoda. BMC Genomics. 2013;14(1):923. https://doi.org/10.1186/1471-2164-14-923.

57. Yin D, Schwarz EM, Thomas CG, Felde RL, Korf IF, Cutter AD, Schartner CM, Ralston EJ, Meyer BJ, Haag ES. Rapid genome shrinkage in a self-fertile nematode reveals sperm competition proteins. Science. 2018;359(6371):5561. https://doi.org/10.1126/science.aao0827.

58. Kanzaki N, Tsai IJ, Tanaka R, Hunt VL, Liu D, Tsuyama K, Maeda Y, Namai S, Kumagai R, Tracey A, Holroyd N, Doyle SR, Woodruff GC, Murase K, Kitazume H, Chai C, Akagi A, Panda O, Ke HM, Schroeder FC, Wang J, Berriman M, Sternberg PW, Sugimoto A, Kikuchi T. Biology and genome of a newly discovered sibling species of Caenorhabditis elegans. Nat Commun. 2018; 9(1):3216. https://doi.org/10.1038/s41467-018-05712-5.

59. Edgar RC. MUSCLE: a multiple sequence alignment method with reduced time and space complexity. BMC Bioinformatics. 2004;5(1):113. https://doi. org/10.1186/1471-2105-5-113.

60. Schliep KP. Phangorn: phylogenetic analysis in R. Bioinformatics. 2011;27(4): 592-3. https://doi.org/10.1093/bioinformatics/btq706.

\section{Publisher's Note}

Springer Nature remains neutral with regard to jurisdictional claims in published maps and institutional affiliations.

\section{Ready to submit your research? Choose BMC and benefit from:}

- fast, convenient online submission

- thorough peer review by experienced researchers in your field

- rapid publication on acceptance

- support for research data, including large and complex data types

- gold Open Access which fosters wider collaboration and increased citations

- maximum visibility for your research: over $100 \mathrm{M}$ website views per year

At BMC, research is always in progress.

Learn more biomedcentral.com/submissions 